\title{
Modelling Functional Requirements in Spatial Design
}

\author{
Mehul Bhatt, Joana Hois, Oliver Kutz, and Frank Dylla \\ SFB/TR 8 Spatial Cognition, University of Bremen, Germany
}

\begin{abstract}
We demonstrate the manner in which high-level design requirements, e.g., as they correspond to the commonsensical conceptualisation of expert designers, may be formally specified within practical information systems, wherein heterogeneous perspectives and conceptual commitments are needed. Focussing on semantics, modularity and consistency, we argue that our formalisation serves as a synergistic interface that mediates between the two disconnected domains of human abstracted qualitative/conceptual knowledge and its quantitative/precisionoriented counterpart within systems for spatial design. Our demonstration utilises simple, yet real world examples.
\end{abstract}

\section{Conceptual Modelling for Spatial Design}

This paper investigates the role of ontological formalisation as a basis for modelling high-level conceptual requirement constraints within spatial design. We demonstrate the manner in which high-level functional requirements, e.g., as they correspond to the commonsensical conceptualisation of expert designers, may be formally specified within practical information systems. Here, heterogeneous perspectives and conceptual commitments are needed for capturing the complex semantics of spatial designs and artefacts contained therein. A key aspect of our modelling approach is the use of formal qualitative spatial calculi and conceptual design requirements as a link between the structural form of a design and the differing functional capabilities that it affords or leads to. In this paper, we focus on the representational modalities that pertain to ontological modelling of structural forms from different perspectives: human / designer conceptualisations and qualitative spatial abstractions suited to spatial reasoning, and geometric primitives as they are applicable to practical information systems for computer-aided design (CAD) in general, and computer-aided architecture design (CAAD) in particular. Our modelling is focussed on semantics, modularity and functional requirement consistency, as elaborated on in the following:

$\triangleright$ Semantics. The expert's design conceptualisation is semantic and qualitative in nature - it involves abstract categories such as Rooms, Doors, Motion Sensors and the spatial (topological, directional, etc.) relationships among them, e.g., 'Room $A$ and Room B have a Door in Between, which is monitored by Camera $C$ '. Professional design tools lack the ability to exploit such design expertise that a designer is equipped with, but unable to communicate to the design tool explicitly in a manner consistent with its inherent human-centred conceptualisation, i.e., semantically and qualitatively. 
$\triangleright$ Modular and Multi-dimensional Representation. An abstraction such as a Room or Sensor may be identified semantically by its placement within an ontological hierarchy and its relationships with other conceptual categories. This is what a designer must deal with during the initial design conceptualisation phase. However, when these notions are transferred to a CAD design tool, the same concepts acquire a new perspective, i.e., now the designer must deal with points, line-segments, polygons and other geometric primitives. Within contemporary design tools, there is no way for a knowledge-based system to make inferences about the conceptual design and its geometric interpretation within a CAD model in a unified manner.

$\triangleright$ Functional Requirements. A crucial aspect that is missing in contemporary design tools is the support to explicitly characterise the functional requirements of a design. For instance, it is not possible to model spatial artefacts such as the range space of a sensory device (e.g., camera, motion sensor), which is not strictly a spatial entity in the form of having a material existence, but needs to be treated as such nevertheless. For instance, consider the following constraint: 'the motion-sensor should be placed such that the door connecting room $A$ and room $B$ is always within the sensor's range space'. The capability to model such a constraint is absent from even the most state-of-the-art design tools.

Organisation of paper. We present an overview of our conceptual modelling approach and the manner in which our formalisation serves as a synergistic interface that mediates between the two disconnected domains of human abstracted qualitative/conceptual knowledge and its quantitative/precision-oriented counterpart within practical information systems. Section 2 presents the concept of ontological modularity and its use for modelling multi-perspective design requirements using a spatial ontology. Section 3 details some key aspects of our spatial ontology and requirements modelled therein. Finally, Section 4 concludes.

\section{Multi-Perspective Representation and Modularity}

Modularity has become one of the key issues in ontology engineering, covering a wide spectrum of aspects (see [11]). The main research question is how to define the notion of a module and how to re-use such modules.

\subsection{Ontological Modules}

The architectural design process defines constraints of architectural entities that are primarily given by spatial types of information. Space is particularly defined from a conceptual, qualitative, and quantitative perspective. The three ontological modules are briefly discussed in the following:

M1 - Conceptual Module. This ontological module reflects the most general, or abstract, terminological information concerning architectural entities: they are conceptualised according to their essential properties, i.e., without taking into account the possible contexts into which they might be put. The ontology 


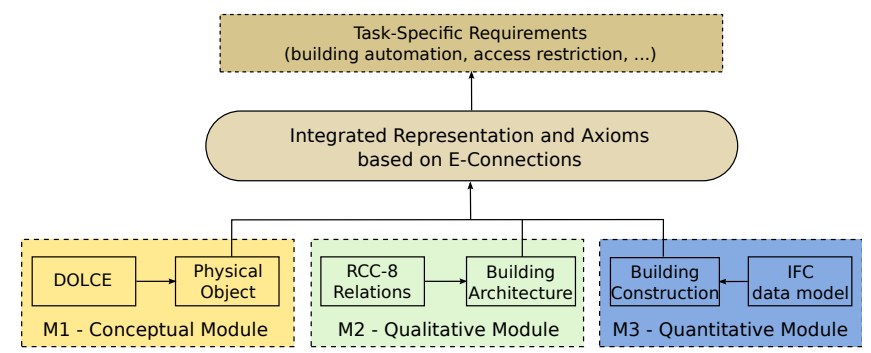

Fig. 1: Multi-Dimensional Representation

Physical Object categorises these entities with respect to their attributes, dependencies, functional characteristics, etc. It is based on DOLCE [9].

M2 - Qualitative Module. This module reflects qualitative spatial information of architectural entities. It specifies the architectural entities based on their regionrelated spatial characteristics. ${ }^{1}$ In particular, the ontology uses relations as provided by the RCC- 8 fragment of the Region Connection Calculus (RCC) [10]. Here, we reuse an RCC ontology that has been introduced in [6], which defines the taxonomy for RCC- 8 relations.

M3 - Quantitative Module. This ontological module reflects metrical and geometric information of architectural entities, i.e., their polygon-based characteristics in the floor plan. It is closely related to an industrial standard for data representation and interchange in the architectural domain, namely the Industry Foundation Classes (IFC) [5]. This quantitative module specifies those entities of the architectural domain that are necessary to describe structural aspects of environments. Especially, information that is available by construction plans of buildings are described here.

\section{$2.2 \mathcal{E}$-Connecting Multiple Perspectives}

The main aspects of modularity are: syntactic and logical heterogeneity, notions of module, distributed semantics and modular reasoning. Here, we restrict ourselves to describing our use of $\mathcal{E}$-connections for multi-perspective modelling of spatial or architectural design.

In order to model spatial design scenarios, we need to be able to cover rather disparate aspects of 'objects' on various conceptual (and spatial) levels. $\mathcal{E}$-connections allow a formal representation of different views on the same domain together with a loose coupling of such views by means of axiomatic constraints employing so-called 'link-relations' to formally realise the coupling. Specifically, in $\mathcal{E}$-connections, an 'abstract' object $o$ of some description logic (DL) can, e.g., be related via a relation $E$ to its spatial extension in a logic such as RCC-8 (i.e.

\footnotetext{
${ }^{1}$ We concentrate on region-based spatial relations, as they are most suitable for our architectural design examples. However, other spatial relations (e.g., for distances, shapes, or orientations) may be applied as well.
} 


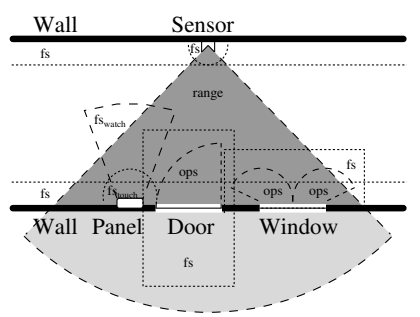

Fig. 2: Concrete Interpretations in $\mathbb{R}^{2}$

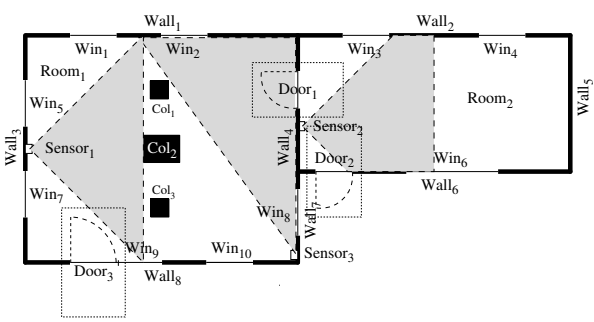

Fig. 3: Spatial Artefact Groundings

a (regular-closed) set of points in a topological space), by a relation $T$ to its life-span in a temporal logic (i.e. an interval of time points), or by a relation $S$ to another conceptual view (i.e. the concept of all rooms object $o$ may be found in). Essentially, the language of an $\mathcal{E}$-connection is the (disjoint) union of the original languages enriched with operators capable of talking about the link relations (see [8] for technical details).

The connection of the three modules (M1-M3) is formalised by axiomatising the used link relations. The Integrated Representation defines couplings between classes from different modules. An overall integration of these thematic modules is achieved by $\mathcal{E}$-connecting the aligned vocabulary along newly introduced link relations and appropriate linking axioms. Based on this Integrated Representation, the module for task-specific requirements specifies additional definitions and constraints to the architectural information available in the modules (M1M3). It formulates requirements that describe certain functions that a specific design, e.g. a concrete floor plan, has to satisfy. They can codify building regulations that a work-in-progress design generally must meet, as explained next.

\section{Functional Requirement Constraints in Architecture}

Semantic descriptions of designs and their requirements acquires real significance when the spatial and functional constraints are among strictly spatial entities as well as abstract spatial artefacts. This is because although spatial artefacts may not be physically extended within a design, they need to be treated in a real physical sense nevertheless. In general, architectural working designs only contain physical entities. Therefore, it becomes impossible for a designer to model constraints involving spatial artefacts at the design level. In [1], we identified three important types of spatial artefacts (this list is not assumed to be exhaustive):

A1. the operational space denotes the region of space that an object requires to perform its intrinsic function that characterises its utility or purpose

A2. the functional space of an object denotes the region of space within which an agent must be located to manipulate or physically interact with a given object

A3. the range space denotes the region of space that lies within the scope of a sensory device such as a motion or temperature sensor

Fig. 2 provides a detailed view on the different kinds of spaces we introduced. From a geometrical viewpoint, all artefacts refer to a conceptualised and derived 


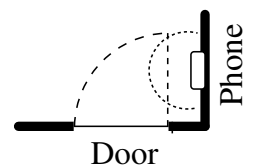

(a) Door 1

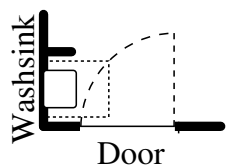

(b) Door 2

Fig. 4: Killer Doors.

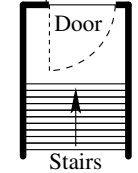

(a) Consistent

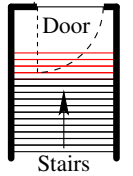

(b) Inconsistent

Fig. 5: Building code for doors and upward stairs-Landesbauordnung Bremen $\S 35(10)$.

physical spatial extension in $\mathbb{R}^{n}$. The derivation of an interpretation may depend on object's inherent spatial characteristics (e.g., size and shape), as well as additional parameters referring to mobility, transparency, etc. We utilise the spatial artefacts introduced in (A1-A3) towards formulating functional requirements constraints for a work-in-progress spatial design. (C1-C2) may need to be satisfied by a design:

C1. Steps of a staircase may not be connected directly to a door that opens in the direction of the steps. There has to be a landing between the staircase steps and the door. The length of this landing has to have at least the size of the door width. ("Bremen building code"/Landesbauordnung Bremen $§ 35$ (10))

C2. People should not be harmed by doors opening up. In general, the operation of a door should be non-interfering with the function / operation of surrounding objects.

Constraints such as $(\mathrm{C} 1-\mathrm{C} 2)$ involve semantic characterisations and spatial relationships among strictly spatial entities as well as other spatial artefacts. In Fig. 5 we depict a consistent and an inconsistent design regarding this requirement. This official regulation can be modelled in the integrated representation by using the link relations introduced in Section 2. The regulation is specified by the ontological constraint that no operational space of a door is allowed to overlap with the steps of a staircase:

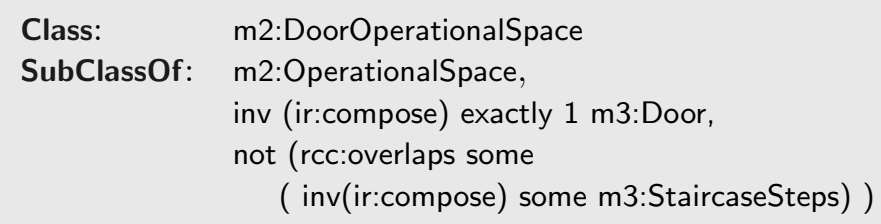

In this example, the different modules are closely connected with each other. In detail, categories in the qualitative modules M2, namely DoorOperationalSpace which is a subclass of OperationalSpace, are related to entities in the quantitative module M3, namely Door and StaircaseSteps, by the link relations given in the integrated representation module, namely compose.

\section{Conclusion}

The work described in this paper is part of an initiative that aims at developing the representation and reasoning methodology [2] and practically usable tools [4] 
for intelligent assistance in spatial design tasks. We have provided an overview of the overall approach to encoding design semantics within an architectural assistance system. High-level conceptual modelling of requirements, and the need to incorporate modular specifications therein, were the main topics covered in the paper. Because of parsimony of space, we could only provide a glimpse of the representation; details of the formal framework and ongoing work may be found in $[3,7]$.

Acknowledgements. We acknowledge the financial support of the DFG through the Collaborative Research Center SFB/TR 8 Spatial Cognition. Mehul Bhatt also acknowledges funding by the Alexander von Humboldt Stiftung, Germany. Participating projects in this paper include: [DesignSpace], I1-[OntoSpace] and R3-[Q-Shape]. We thank Graphisoft - http://www.graphisoft.com/ - for providing licenses for the design tool ArchiCAD v13 2010.

\section{Bibliography}

[1] M. Bhatt, F. Dylla, and J. Hois. Spatio-terminological inference for the design of ambient environments. In K. S. Hornsby, C. Claramunt, M. Denis, and G. Ligozat, editors, Conference on Spatial Information Theory (COSIT'09), pages 371-391. Springer-Verlag, 2009.

[2] M. Bhatt and C. Freksa. Spatial computing for design: An artificial intelligence perspective. In NSF International Workshop on Studying Visual and Spatial Reasoning for Design Creativity (SDC'10), 2010. (to appear). http://www.cosy. informatik. uni-bremen.de/staff/bhatt/seer/Bhatt-Freksa-SDC-10.pdf.

[3] M. Bhatt, J. Hois, and O. Kutz. Modelling Form and Function in Architectural Design. 2010. Submitted to a journal, http://www. cosy.informatik.uni-bremen.de/staff/ bhatt/seer/form-function.pdf.

[4] M. Bhatt, A. Ichim, and G. Flanagan. DSim: A Tool for Assisted Spatial Design. In Proceedings of the 4 th International Conference on Design Computing and Cognition (DCC'10), 2010.

[5] T. Froese, M. Fischer, F. Grobler, J. Ritzenthaler, K. Yu, S. Sutherland, S. Staub, B. Akinci, R. Akbas, B. Koo, A. Barron, and J. Kunz. Industry Foundation Classes for Project Management-A Trial Implementation. ITCon, 4:17-36, 1999. www.ifcwiki.org/.

[6] R. Grütter, T. Scharrenbach, and B. Bauer-Messmer. Improving an RCCDerived Geospatial Approximation by OWL Axioms. In ISWC-08, pages 293-306. Springer, 2008.

[7] J. Hois, M. Bhatt, and O. Kutz. Modular Ontologies for Architectural Design. In Proc. of the 4th Workshop on Formal Ontologies Meet Industry, FOMI-09, Vicenza, Italy, volume 198 of Frontiers in Artificial Intelligence and Applications, pages 66-77. IOS Press, 2009.

[8] O. Kutz, C. Lutz, F. Wolter, and M. Zakharyaschev. $\mathcal{E}$-Connections of Abstract Description Systems. Artificial Intelligence, 156(1):1-73, 2004.

[9] C. Masolo, S. Borgo, A. Gangemi, N. Guarino, and A. Oltramari. WonderWeb Deliverable D18: Ontology Library. Technical report, ISTC-CNR, 2003.

[10] D. A. Randell, Z. Cui, and A. G. Cohn. A spatial logic based on regions and connection. In Proc. of KR-92, pages 165-176, 1992.

[11] H. Stuckenschmidt, C. Parent, and S. Spaccapietra, editors. Modular Ontologies, volume 5445 of $L N C S$. Springer, 2009. 